\title{
ROLE OF PHOSPHOFRUCTOKINASE IN THE REGULATION OF THE SUBSTRATE CYCLE IN THE BUMBLE BEE Bombus atratus
}

\author{
LEITE, A. ${ }^{1}$; CRIVELLARO, $\mathrm{O}^{2}$ \\ ${ }^{1}$ Centro de Biologia Molecular e Engenharia Genética, Universidade Estadual de Campinas; \\ ${ }^{2}$ Departamento de Medicina, Pontifícia Universidade Católica do Paraná. \\ Kinetic studies carried out with phosphofructokinase (PFK) purified from the flight \\ muscle of the bumble bee Bombus atratus displayed two possible sequential \\ mechanisms, ordered $\mathrm{BiBi}$ or random $\mathrm{BiBi}$, as described by Cleland. The allosteric \\ regulatory properties of PFK were studied at $\mathrm{pH} 7,4$. To study the role of PFK in the \\ control of the F6P/F1,6BP substrate cycle the concentration of several intermediate \\ metabolites involved in the metabolic control of PFK from the flight muscle of the \\ bumble-bee were assayed under physiological conditions which affect the activity of \\ both glycolysis and the substrate cycle. The results indicate that the activity of the \\ glycolytic pathway in the substrate cycle in the flight muscle of bumble bee is solely \\ possible in the presence of potent modulators of PFK such as F2,6BP and AMP.
}

Key Words: Phosphofructokinase, PFK, fructose 2,6-bisphosphate, F2,6BP, substrate cycle, Bumble-bee. 